\title{
Neuro-Fuzzy Dual-Mode Control of Travelling-Wave Ultrasonic Motors
}

\author{
S.W. Chung, K.T. Chau, and C.C. Chan \\ Department of Electrical and Electronic Engineering, The University of Hong Kong, Pokfulam Road, Hong Kong
}

\begin{abstract}
With their significant advantages over traditional electromagnetic motors, ultrasonic motors (USMs) are becoming attractive. This paper presents a neuro-fuzzy dualmode controller, combining the knowledge-based fuzzy logic and the learning-incorporated neural network as well as using dual control inputs, for servo control of travelling-wave USMIs. According to the proposed neuro-fuzzy control, both the driving frequency and phase difference of the USM system are simultaneously on-line adjusted. Experimental results have verified its superior steady-state and dynamic performances under various reference inputs.
\end{abstract}

\section{INTRODUCTION}

Owing to their low-speed-high-torque characteristics, compact size, quiet operation, fast response, inherent holding torque and free of electromagnetic interference, ultrasonic motors (USMs) have quickly received significant attention. Particular interests have recently been laid on the travellingwave type because of its unique hollow structure and longer life span. However, it exhibits heavily nonlinear control characteristics, and its parameters are also very sensitive to changes in temperature. Under such circumstances, various kinds of empirical control approaches, such as fuzzy logic control, adaptive control and neural network control, have been proposed for position control of this USM [1]-[3]. To solicit the above strengths and suppress their deficiencies, it is the purpose of this paper to newly introduce the neurofuzzy control (NFC) for travelling-wave USMs using dual control inputs - the phase difference and driving frequency.

\section{TRAVELLING-WAVE USM}

An USM is a type of actuator that uses mechanical vibrations in the ultrasonic range (over $20 \mathrm{kHz}$ ) as its driving source. The operating principle of the travelling-wave USM is fundamentally different from all electromagnetic motors. Fig. 1 illustrates its basic operating principle. Their typical nonlinear characteristics are illustrated in Fig. 2. As shown in Fig. 2, the relationships between rotating speed $N$ and driving frequency $f$ as well as between $N$ and phase difference $\phi$ are highly nonlinear and seriously dependent on the load torque. Moreover, these operating characteristics are sensitive to the motor temperature, hence resulting an additional timevarying nonlinearity.

\section{NEURO-FuZZY DUAL-MODE CONTROL}

Recently, a number of NFC schemes have been proposed, focusing on the variations of structure and parameter learning. Fig. 3 shows the NFC structure for the proposed servo position control of travelling-wave USMs. In this 5layered structure, the nodes in the layer 1 (called the input layer) simply direct the crisp input states (namely the position error $\varepsilon$ and motor speed $\omega$ ) to the layer 2 . The nodes in the layer 2 are input term nodes representing the input linguistic variables. They act as membership functions to transform those crisp values into fuzzy numbers, so-called fuzzification. Each node in the layer 3 is a rule node that represents one fuzzy logic rule. Hence, all the layer- 3 nodes form a fuzzy rule base. The nodes in the layer 4 are output term nodes representing the output linguistic variables. They serve to integrate the fired rules which have the same consequent. Finally, the nodes in the layer 5 (called the output layer) convert the resulting fuzzy numbers into the crisp output control signals (the frequency change $\Delta f$ and phase-difference change $\Delta \phi$ ), so-called defuzzification. The corresponding fuzzy control rules for the linguistic $\Delta f$ and $\Delta \phi$ with respect to the linguistic $\varepsilon$ and $\omega$ are summarized in Table $I$.

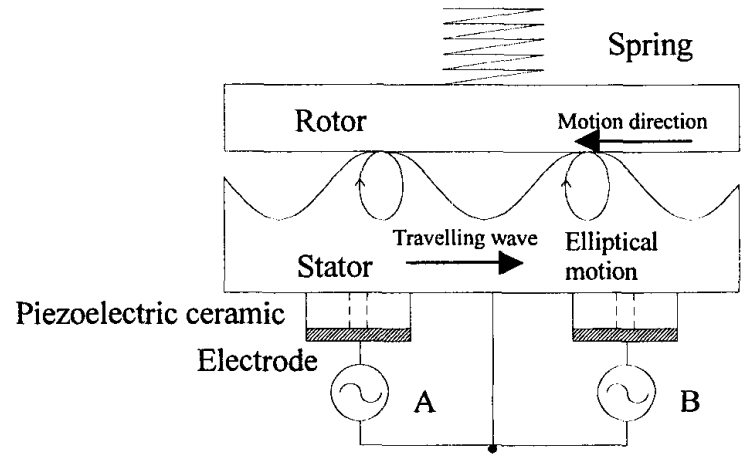

Fig. 1. USM operating principle.

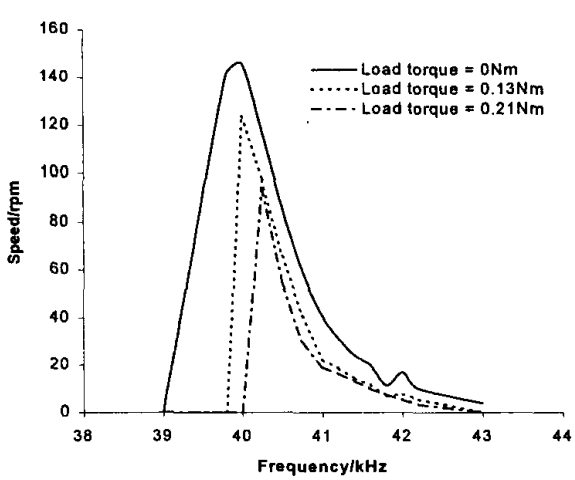

(a) 


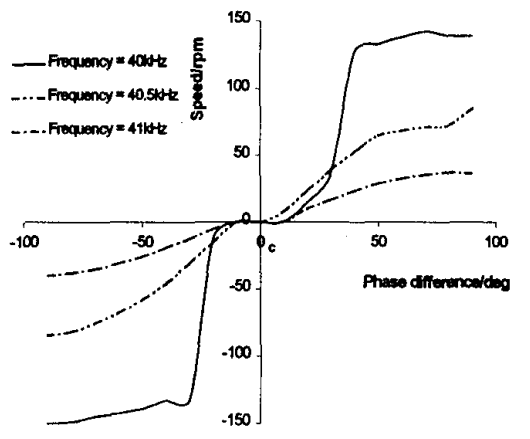

(b)

Fig. 2. USM characteristics. (a) $N$ vs $f$. (b) $N$ vs $\phi$.

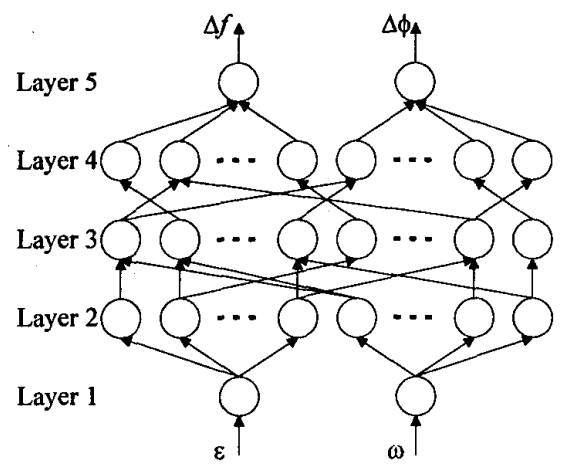

Fig. 3. NFC structure.

TABLE I. FUZZY CONTROL RULES. (a) $\Delta f$. (b) $\Delta \phi$.

\begin{tabular}{|c|c|c|c|c|c|c|c|}
\hline$\varepsilon \backslash \omega$ & NB & NM & NS & ZO & PS & PM & PB \\
\hline NB & ZO & NS & NM & NB & NM & NS & ZO \\
\hline NM & PS & ZO & NS & NM & NS & ZO & PS \\
\hline NS & PM & PS & ZO & NS & ZO & PS & PM \\
\hline ZO & PB & PM & PS & PB & PS & PM & PB \\
\hline PS & PM & PS & ZO & NS & ZO & PS & PM \\
\hline PM & PS & ZO & NS & NM & NS & ZO & PS \\
\hline PB & ZO & NS & NM & NB & NM & NS & ZO \\
\hline \multicolumn{7}{|c|}{} \\
\hline$\varepsilon \backslash \omega$ & NB & NM & NS & ZO & PS & PM & PB \\
\hline NB & ZO & PS & PS & PB & PS & PS & ZO \\
\hline NM & NS & ZO & PS & PB & PS & ZO & NS \\
\hline NS & NB & NS & ZO & PS & ZO & NS & NB \\
\hline ZO & NB & NB & NS & ZO & NS & NB & NB \\
\hline PS & NB & NS & ZO & PS & ZO & PS & PM \\
\hline PM & NS & ZO & PS & PB & PS & ZO & NS \\
\hline PB & ZO & PS & PS & PB & PS & PS & ZO \\
\hline
\end{tabular}

\section{SYSTEM SET.UP}

As shown in Fig. 4, the power stage circuit is composed of the 2-phase inverter and the USM. This inverter operates at a high switching frequency, nominally at $40 \mathrm{kHz}$. The corresponding dc supply voltage is of $90 \mathrm{~V}$. Those power devices connecting to the lower rail are n-channel IRF740 MOSFET and those to the upper rail are p-channel IRF9640 MOSFET so that the required number of isolated dc supplies for gate driving can be minimized. Since the USM is inherently capacitive, inductors are added in series to its terminals so that the inverter can offer the advantages of soft switching - reduction of switching loss and electromagnetic interference (EMI).

In order to assess the performance of the proposed NFC approach for servo position control, a PI dual-mode controller is adopted for comparisons. This dual-mode controller applies PI control $\left(K_{i}=0.008\right.$ and $\left.K_{p}=0.5\right)$ to adjust the phase difference whereas only proportional control $\left(K_{p}=0.05\right)$ for frequency adjustment. These empirical parameters are chosen by trial and error in such a way that the optimal performance occurs at rated conditions.

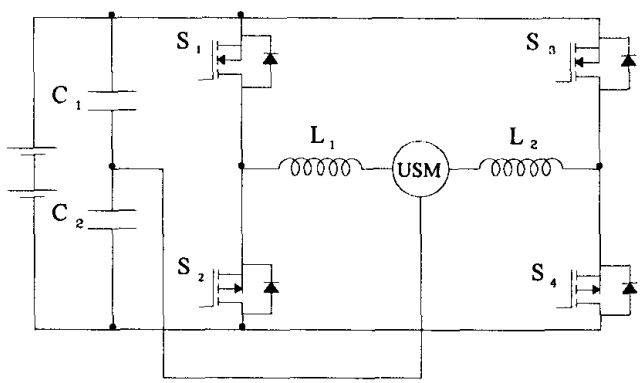

Fig. 4. USM power stage circuit.

\section{RESULTS AND COMPARISONS}

Fig. 5 shows the position responses of the USM when the controller adopts the dual-mode control. For the case that the position command is of a square wave, the USM fails to offer good steady-state accuracy. Also, for the case of a sinusoidal command, it suffers from significant position errors at both positive and negative peaks. In contrast, when the proposed NFC approach is used, the corresponding position responses shown in Fig. 6 are much better. Upon a square-wave command, the NFC controller can produce a near-zero steady-state error; whereas upon a sinusoidal command, the USM can accurately track the command even at both the positive and negative peaks. As expected, the initial position responses using NFC are worse than those using dual-mode control because the NFC controller is initially immature whereas the dual-mode controller has been empirically tuned. Nevertheless, the NFC controller can quickly learn from successive operation so that the USM can accurately track the commands within $4 \mathrm{~s}$. Moreover, during the tracking of the sinusoidal command, a load torque of $0.13 \mathrm{Nm}$ is suddenly applied to the USM at the time instant of $30 \mathrm{~s}$. Fig. 6 (b) 
shows that there is no observable change in the position response, indicating that the NFC approach can offer the position response immune to the change of load torque.

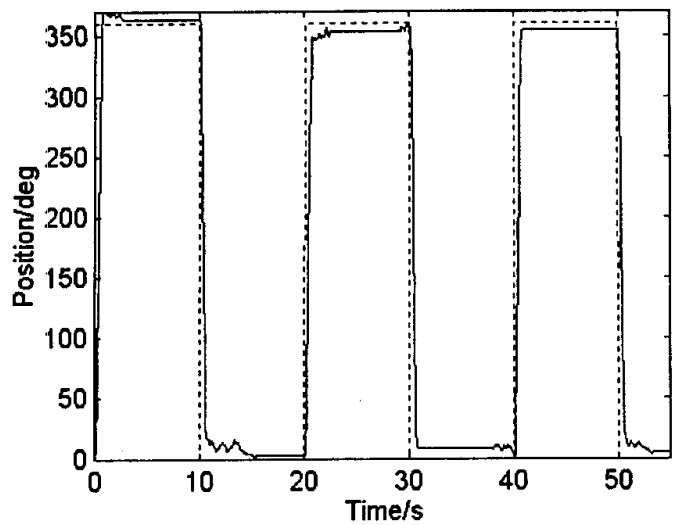

(a)

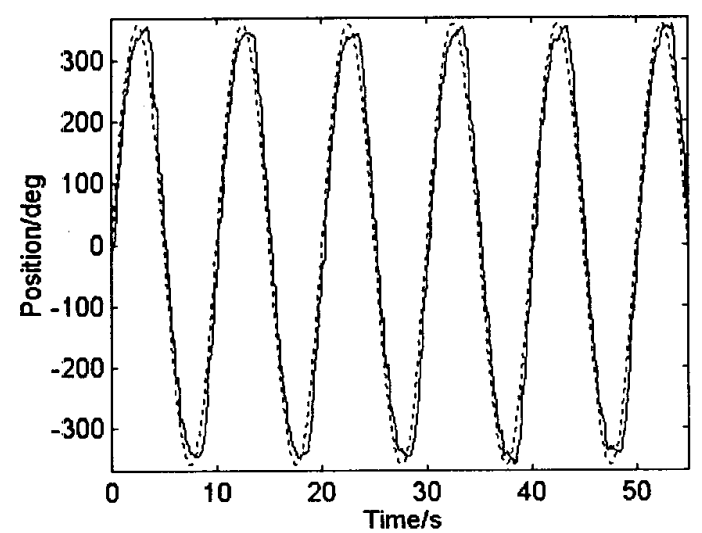

(b)

Fig. 5. Position responses of PI dual-mode control. (a) Square-wave command. (b) Sinusoidal command.

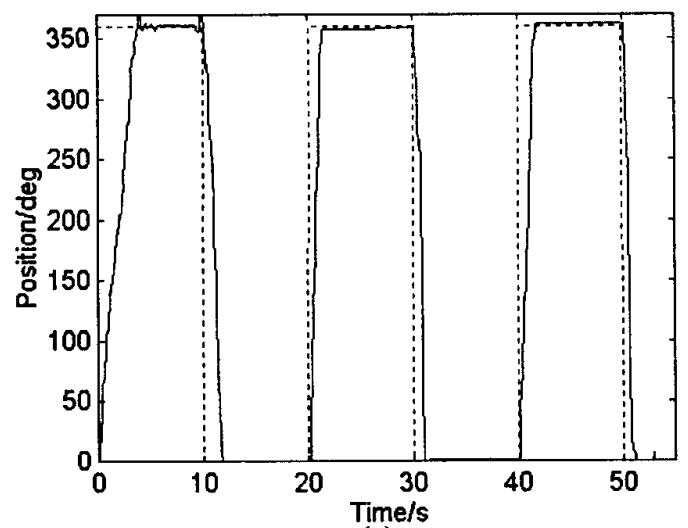

(a)

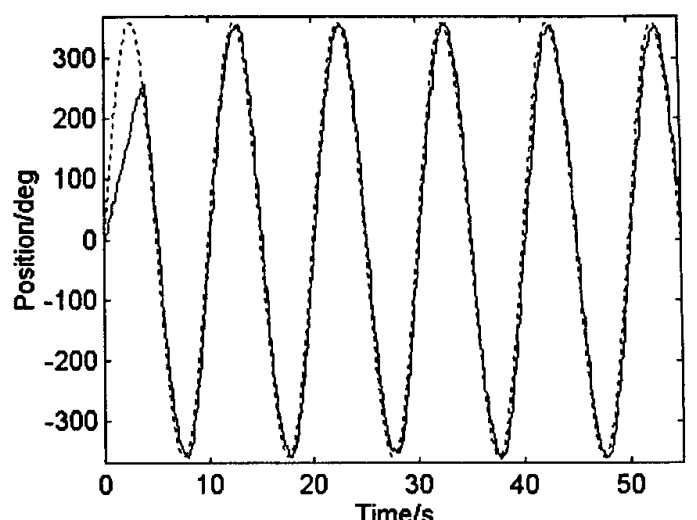

(b)

Fig. 6. Position responses of NFC. (a) Square-wave command. (b) Sinusoidal command.

\section{CONCLUSION}

In this paper, a neuro-fuzzy dual-mode controller has been successfully developed for servo position control of the travelling-wave USM. This controller integrates both the strengths of fuzzy logic control and neural network control, namely the expert knowledge for USM control based on the fuzzy logic realization, and the on-line adjustment of the controller due to the neural network structure. Compared to the PI dual-mode controller, the proposed neuro-fuzzy controller offers much better performance for tracking both square-wave and sinusoidal commands. Moreover, its superior performance on the robustness to the changes of load torque and command pattern are demonstrated. Since the proposed approach is so general, it can readily be applied to all other types of USMs.

\section{ACKNOWLEDGMENT}

This work was supported and funded in part by the CRCG of the University of Hong Kong, and the Hong Kong Research Grants Council.

\section{REFERENCES}

[1] S.I. Furuya, Y. Ohkura and T. Maruhashi, "A novel inverter-drive ultrasonic motor-actuated positioning servo motion system using a fuzzy reasoning control scheme," Journal of Circuits, Systems and Computers, Vol. 4, No. 4, pp. 395-414, 1994.

[2] F.J. Lin, "Fuzzy adaptive model-following position control for ultrasonic motor," IEEE Transactions on Power Electronics, Vol. 12, No. 2, pp. 261-268, 1997.

[3] T. Senjyu, H. Miyazato, and K. Uezato, "Position control of ultrasonic motors using neural network," IEEE International Industrial Electronics Symposium, pp. 368$373,1996$. 